\title{
De la recherche empirique à l'analyse sociologique
}

Les conditions sociales de la production académique

\section{Avertissement}

Le contenu de ce site relève de la législation belge sur la propriété intellectuelle et est la propriété exclusive de l'éditeur.

Les œuvres figurant sur ce site peuvent être consultées et reproduites sur un support papier ou numérique sous réserve qu'elles soient strictement réservées à un usage soit personnel, soit scientifique ou pédagogique excluant toute exploitation commerciale. La reproduction devra obligatoirement mentionner l'éditeur, le nom de la revue, l'auteur et la référence du document.

Toute autre reproduction est interdite sauf accord préalable de l'éditeur, en dehors des cas prévus par la législation en vigueur en Belgique.

\section{Référence électronique}

Magali Ballatore (2011) « De la recherche empirique à l'analyse sociologique », Émulations, janvier 2011.

URL : http://www.revue-emulations.net/ballatore

Éditeur : Émulations - Revue des jeunes chercheurs en sciences sociales

http://www.revue-emulations.net 


\title{
De la recherche empirique à l'analyse sociologique
}

\author{
Les conditions sociales de la production académique
}

\author{
Magali Ballatore \\ Chercheure à I' Institute of Analysis of Change in Contemporary and \\ Historical Societies (IACCHOS) \\ Université catholique de Louvain
}

\begin{abstract}
Les individus, comme les chercheurs eux-mêmes, évoluent simultanément dans tous les registres de l'action et de la pratique. Cet article est une contribution à la réflexion sur les fondements d'un raisonnement sociologique en perpétuel changement. Il ouvre des pistes pour tenter de répondre aux questions posées par Claude Dubar en 2006 « Quelles relations entre les " positions et prises de position » sociales et idéologiques, le statut d'emploi et le type de sociologie pratiquée ? Il est également une incitation à sortir de la tendance au classement des objets dans des cases paradigmatiques étanches, à s'intéresser collectivement à nos propres parcours et pratiques professionnelles, à ce qui définit l'activité de connaissance et les procédures concrètes qui la réalisent. Il veut aussi rappeler, comme bien d'autres l'on fait antérieurement, que par delà ses clivages, les conflits et les discontinuités dans les carrières des enseignants-chercheurs, la sociologie s'organise bien selon « un maillage cognitif », une toile, « des lignes de force ", qui en font une discipline scientifique à part entière (Berthelot, 1990).
\end{abstract}

Peut-on comprendre les évolutions de la discipline sociologique sans s'intéresser à ceux qui la questionnent et aux conditions de la production académique ? Peut-on traiter à la fois, du système et des individus, de la sociologie et des sociologues, du changement et de l'ordre, du « micro » et du " macro »? Afficher cette ambition présente de nombreux risques. Le temps et le courage lui étant nécessaires sont socialement distribués et varient en fonction des contextes économiques et sociaux. Lorsque des sociologues tentent de relier, en suivant les trajectoires des acteurs, des phénomènes sociaux étudiés séparément ou les différentes facettes d'une réalité sociale, dont les contours changent dans le temps, l'éclectisme et la réprobation des collègues menacent. Didier Lapeyronnie parle « d'académisme radical » (Lapeyronnie, 2004). George Simmel, que l'on peut difficilement ranger sous un paradigme bien défini, a souvent et est encore parfois vu comme un essayiste dont la pensée manque de rigueur systématique et d'unité (Berthelot, 1990). Ceci parce que la globalité analysée paraît une « totalité » scientifiquement improbable et normativement dangereuse (Dubet, 1994). Il est pourtant possible de comprendre ce choix comme assumé. Voir toutes les facettes d'un phénomène social est autant un choix de méthode, qu'un choix théorique, même si la société ne peut être considérée comme un système intégré. Les individus et les chercheurs eux-mêmes, évoluent simultanément dans tous les registres de la pratique et aucun d'eux ne suffit à fonder un sujet. Pourquoi alors considérer comme des « sauts épistémologiques » des tentatives, à partir de l'empirie, de rendre à la réalité sociale sa complexité ? Le faire risquerait d'aggraver la tendance au classement des objets de la sociologie dans des cases

(C) 2010 Émulations - Revue des jeunes chercheurs en sciences sociales

www.revue-emulations.net 
paradigmatiques étanches. En effet, certains sociologues refusent systématiquement par choix idéologiques, les notions de « détermination, de structure et de domination » et d'autres, celles de "liberté, de contingence, de conjoncture, etc.». Notions qui pourtant ne sont pas en contradiction dans les trajectoires des individus et peuvent s'articuler.

Cet article veut contribuer à la réflexion sur les fondements d'un raisonnement sociologique en perpétuel changement. Il ouvre des pistes pour tenter de répondre aux questions posés par Claude Dubar en 2006 «Quelles relations entre les « positions et prises de position » sociales et idéologiques, le statut d'emploi et le type de sociologie pratiquée ? Comment définir le champ de la sociologie au-delà des «statutaires » ? » (Dubar, 2006). Tout d'abord, à partir des relations associatives et répulsives entre quelques concepts initiaux, dont dépendent toute la structure du raisonnement sociologique et le développement de la recherche empirique, nous analyserons l'intérêt de partir du terrain pour dépasser les oppositions paradigmatiques. Car il n'y a pas d'un coté un domaine de la complexité, qui serait celui de la théorie et, de l'autre, le domaine des choses simples qui serait celui de l'empirie (Berthelot, 1990). Cette dernière est l'univers concret de la théorie. Nous nous pencherons ensuite sur les sociologues de terrain, sur les conditions sociales de la production sociologique, que l'on évoque peu, mais qui permettent de comprendre, en partie, les évolutions paradigmatiques de la discipline. En effet l'univers matériel et social dans lequel évoluent les sociologues de nos jours, est bien différent de celui qui a vu naître la discipline. Les universités et leurs publics ont changé. La demande sociale provenant des secteurs publics et privés de l'économie a aussi évolué. Les outils de traitement des données se sont développés. La recherche appliquée et de court terme s'accroît. En France, le taux de docteurs qui accède à un " poste » d'enseignant-chercheur ou de chercheur dans un organisme public de recherche ne fait que baisser régulièrement (Chenu, 2001). Tout ceci n'est pas sans conséquences sur le développement d'une discipline et d'un métier de sociologue, qui, en renforçant ses liens avec la « demande sociale », argumente ses droits à l'existence tout en répondant à l'injonction d'utilité, pour éviter la marginalisation.

\section{Des oppositions paradigmatiques à la dialectique}

Comment articuler les diverses dimensions de la réalité sociale ? Comment surmonter les oppositions paradigmatiques par une approche dialectique, qui est une intelligence du double sens, considérant à la fois le local et le global (Vallée, 1995) ? Vallée insiste sur la nécessité d'un va et vient, citant la pensée de Pascal: "Je tiens pour impossible de connaitre les parties sans connaitre le tout, non plus que de connaître le tout sans connaître particulièrement les parties" (Pascal, 1670). Un paradigme peut être défini comme un type de relation logique (inclusion, conjonction, disjonction, exclusion) entre un certain nombre de notions ou catégories maittresses. La sociologie s'est développée par la construction de paradigmes et leurs fragmentations progressives, pour aboutir à la diversité des approches que l'on connait aujourd'hui. Les grands paradigmes en sociologie, sont bien des opérations intellectuelles artificielles en vue de rendre intelligible une réalité composée. Je vais donc ici m’intéresser à quatre ensembles d'opposition que l'on retrouve dans les écrits sociologiques, en procédant de manière discontinue, par l'opposition ou la multiplicité de ce qui est en mouvement, et permet d'atteindre un niveau ternaire. Ici j'utiliserai la dialectique comme une technique de raisonnement qui procède par la mise en parallèle de deux concepts opposés, pour tenter de dépasser la contradiction et aboutir à une synthèse.

\subsection{Acteurs/systèmes}

Que faire pour éviter les travers d'un structuralisme sans sujet ou d'un interactionnisme sans structure? L'articulation de logiques sociétales, institutionnelles et personnelles permet une confrontation des paradigmes. Dès l'écriture de mon mémoire de maitrise sur l'expérience scolaire des étudiants en Sciences en France et en Angleterre, jusqu'à la rédaction de ma thèse de doctorat sur la mobilité institutionnalisée en Europe, j’ai voulu afficher cette ambition et encore aujourd'hui lorsque je rédige des articles scientifiques, au risque de me voir reprocher d'avoir un cadre théorique flottant. Lors de la rédaction des résultats d'une étude, le chercheur est toujours face à une nécessité : celle de sa structuration, fonction des dimensions multiples du phénomène social étudié. Par exemple pour mes recherches en sociologie de l'éducation, il s'agissait de structurer l'analyse en fonction des différentes dimensions du système éducatif: sa dimension sélective, productive/ et de diffusion du savoir, sa dimension intégrative, socialisatrice etc. Ce découpage est bien sûr essentiellement opératoire et sert de clarification à une analyse multiniveau. En effet, dans les discours, entre les divers aspects « objectifs» du monde universitaire et les dimensions de

(C) 2010 Émulations - Revue des jeunes chercheurs en sciences sociales

www.revue-emulations.net 
l'expérience étudiante, les liens ne sont pas si clairement établis. Comment comprendre la sélection sans s’intéresser à ce qui est réellement transmis et appris ? Les loisirs et la sociabilité peuvent-ils être totalement détachés des conditions de vie et de travail des étudiants? Pourtant nous les avons distingués pour une analyse sociologique distincte. De même les programmes d'études et l'hospitalité des lieux dans différents pays ne sont pas sans relation avec les mécanismes sélectifs, mais ce lien n'est presque jamais établi pragmatiquement. Il semble donc peu opératoire de traiter séparément la structure (universitaire) et les activités structurantes (celles des étudiants). Valoriser les situations, les interactions et les processus parait beaucoup plus fécond. Ceci amène à se centrer sur l'acteur, mais cet intérêt ne signifie pas une négligence des contraintes structurelles qui existent dans tout environnement (le milieu scolaire n'étant pas une exception).

Rechercher un niveau intermédiaire d'organisation des pratiques qui trouve sa pertinence dans des configurations d'articulation possibles entre l'acteur et le système, entre logique individuelle et collective semble la voie la moins réductrice pour la compréhension des comportements sociaux. L'antagonisme n'est bien souvent que de la complémentarité. Si l'expérience des agents est socialement produite, elle produit également les pratiques, puisqu'elle contribue à les construire. Rendre compte à la fois des processus d'institutionnalisation et des processus biographiques permet ainsi de lier des dynamiques macro-sociales et le point de vue des acteurs pour reprendre les termes utilisés par Claude Dubar en pensant en quelque sorte une dialectique individu / société qui ne soit pas une opposition (Dubar, 2006).

Le sociologue «pluraliste » qui choisi cette posture analysera alors des trajectoires d'individus, qui participent à un moment donné, par l'intermédiaire d'institutions particulières, de vecteurs, à un même «événement ", à une même « aventure ». Cette dernière peut-être par exemple un cursus scolaire, un séjour d'étude à l'étranger (vecteur scolaire), une mise en couple, une naissance (vecteur familial), un début de carrière, une mobilité sociale (vecteur professionnel). Ces «aventures humaines» peuvent survenir simultanément, en léger ou fort décalage et s'influencer, sans que leurs conséquences se fassent sentir immédiatement. Il est donc toujours délicat de faire transparaître de façon évidente ce qui fait lien et ce qui divise lorsque l'on regarde des trajectoires individuelles et collectives. Le manque de positionnement, de visibilité d'une colonne vertébrale dans certaines études sociologiques peut donc être la conséquence d'une volonté de comprendre un champ de pratiques sous des angles différents, plutôt que de décrire une situation à un temps T par un vecteur unique. C'est en fait un choix d'organisation des idées et des données dans le but de préserver le caractère unitaire du phénomène étudié, qui peut donner une impression de dispersion aux chercheurs dont la pensée est pré-structurée par les paradigmes dominants.

Les références, les catégories d'analyse et les concepts utilisés dans une démarche ouverte, qui recherche l'articulation plutôt que les oppositions stériles, sont donc multiples. Ce qui ne signifie pas un renvoi, un va et vient, sans raisonnement d'un concept à l'autre, mais une structuration par dimension des catégories d'analyse, qui restitue le contexte de leurs naissances respectives. Dans notre étude sur la mobilité Européenne institutionnalisée, l'entrée était bien les trajectoires des jeunes Erasmus, mais par leur intermédiaire, il s'agissait d'étudier des systèmes éducatifs et leurs transformations et les liens entre les institutions éducatives et d'autres espaces institutionnalisés de la vie sociale en Europe. Il s'agissait d'articuler empiriquement un processus et des dimensions d'analyse. Choisir cette entrée portait en soi la volonté assumée d'affiliation à une entreprise de pensée sociologique commune, mais plurielle.

Il n'y a jamais un seul élément qui permette de faire le lien et ordonner de manière univoque les différentes dimensions de la réalité sociale. L'ensemble des laboratoires par lesquels je suis passée en France et à l'étranger expérimente de façon récurrente la difficulté à trouver ce qui fait, dans les axes et séminaires de leur laboratoire, cohérence, « ossature », car une forte cohésion ne peut se faire sans réductionnisme. L'expression de « toile 》 (plutôt que de «fil conducteur»), qui maintient la cohérence et facilite la lecture me semble intéressante. Car, pour l'intelligibilité, il est toujours néanmoins utile de faire des choix, de sélectionner des axes pour penser un phénomène social. Jean Michel Berthelot (Berthelot, 1990) parle de «lignes de saillance de l'objet », « d'espace de pertinence » c'est-à-dire de constellations particulières d'événements qu'un point de vue disciplinaire élabore en structurations pertinentes. Le programme Erasmus se développe à la fois sur des territoires et concerne une population particulière, il est cadre matériel et unité de vie collective, configuration d'objets, de services et nœud de relations entre acteurs sociaux. On peut se pencher plus particulièrement sur l'un ou l'autre de ces ordres de réalités, mais ils n'en demeurent pas moins indissociables. Ainsi la «population» Erasmus, peut être regardée d'abord sous l'angle de la sélection, de la segmentation, puis sous l'angle des ses positions et pratiques dans l'espace, de ses relations et enfin sous l'angle des trajectoires et parcours d'insertion professionnelle, familiale, citoyenne etc. Et chacun de ces axes 
peut utiliser un arsenal méthodologique varié, traverser les autres domaines d'analyse et se constituer en point de vue pertinent pour l'ensemble du phénomène des mobilités institutionnalisées.

\subsection{Autonomie/contrainte}

En mettant en évidence la contribution des différents acteurs dans l'engendrement, la persistance ou le changement des états du système (universitaire par exemple), l'une des difficultés majeures auxquelles doit faire face le sociologue est de déterminer la part respective des libertés des acteurs et des déterminations qui les contraignent (Crozier, Friedberg, 1977). La notion d'autonomie est complexe puisqu'elle dépend de conditions culturelles et sociales. L'autonomie se nourrit de dépendance ; nous dépendons d'une éducation, d'un langage, d'une culture, d'une société. Nous dépendons aussi d'ailleurs de capacités physiologiques, elles-mêmes produites d'un programme génétique. Si on prend l'exemple du séjour à l'étranger, comment faire la part respective de ce qui tient à de la contingence, à de l'autonomie dans le choix des étudiants et de ce qui tient à des situations socioéconomiques et politiques qui influencent les orientations? Le séjour Erasmus (et plus largement l'éducation) reproduit la société, qu'il reflète autant et peut-être davantage qu'il ne la transforme, même si les acteurs locaux ont une autonomie relative, qu'il ne faut ni surestimer, ni sous-estimer. L'actuelle mobilité étudiante et professionnelle n'est jamais la résultante de facteurs d'attraction et de répulsion détachés d'intermédiaires, de médiateurs et de rapports de pouvoir humain localisés. Même si certains acteurs de la mondialisation se plaisent à souligner leur entière liberté ou autonomie, beaucoup sont tributaires des conditions qui leur ont été offertes. De même, la variabilité de leur degré de manœuvre dépend en grande partie de leurs origines nationales et disciplinaires et par la suite du type de vie, notamment professionnelle, dans laquelle ils se seront engagés.

Les jeunes Erasmus participent à un programme, tout en développant des stratégies. Or, la notion de stratégie s'oppose à celle de programme. "Le programme est institué et instituant » (Morin 1994 : 119). Une stratégie se détermine en tenant compte d'une situation aléatoire, d'éléments adverses, et elle est amenée à se modifier en fonction d'informations changeantes. "Tous les acteurs n'ont qu'une liberté restreinte et ne sont capables corrélativement que d'une rationalité limitée » (Crozier \& Friedberg 1977 : 55). Les stratégies étudiantes introduisent de la souplesse, des inattendus dans un programme institutionnalisé. Mais il faut voir également, qu'une organisation, comme l'université, peut aussi adopter ou développer un programme de manière stratégique, mais dans les organisations, il n'y a pas que des stratèges ou stratagèmes, certains choix peuvent être faits en toute naïveté ou sans connaissance de cause. Toute expérience sociale a une dimension stratégique, mais aucune ne s'y réduit. Elle appelle aussi à l'irrationalité des racines, des appartenances et des liens et à celle des convictions ou de la recherche d'une authenticité plus ou moins improbable.

Nous avons constaté dans nos recherches, que, même si parler de «brain movement » permet de s'éloigner d'une conception négative des migrations, en Italie par exemple, la part de la force de travail qualifiée croît de façon continue par rapport au total des émigrés italiens et le départ est souvent corrélé à une fuite de conditions et/ou d'insertion sur le marché du travail difficiles et d'une certaine situation politique et sociale. Finalement la mobilité est la face positive de l'émigration. Car les migrations par le passé comme aujourd'hui, sont souvent l'expression d'une recherche de meilleures conditions de vie. Il apparaît difficile de faire la part entre ce qu'il y a de positif en terme d'émancipation, d'autonomie, de choix et de ce qu'il y a de plus négatif en terme de fuite, de contrainte dans ces nouveaux mouvements migratoires, peut-être moins définitifs, mais qui laissent plus longtemps des individus dans un « entre-multiples lieux ». De nombreux auteurs distinguent pourtant les migrations passées et actuelles comme étant, pour les premières forcées et pour les secondes résultantes d'un choix libre et autonome.

Les jeunes italiens qui aujourd'hui se trouvent au Royaume-Uni seraient le fruit de deux modèles migratoires différents : Dans le premier on trouve les fils ou petit-fils des générations passées de migrants, qui ont quitté l'Italie dans les années d'après-guerre, dans le second, les auteures classent au contraire les migrants professionnels, " expression de la nouvelle émigration et donc artisan d'un choix plus autonome (par rapport au passé) de vivre à l'étranger. »[1] (Bartolini \& Volpi 2005 : 93). Ce groupe de «neo-immigrati » se compose de personnes possédant des niveaux élevés de qualification, motivés par les opportunités de carrière que le Royaume-Uni leur offre. Tout le reste du propos de ces auteures est en contradiction avec l'affirmation d'un libre arbitre. Elles parlent ainsi des critiques que ces migrants adressent à leur pays en termes d'opportunités professionnelles. Plus la permanence se prolonge, plus l'insatisfaction de ces jeunes semble forte. Lorsqu'ils réfléchissent à un éventuel retour en Italie ou vers le sud de l'Europe, les champs du possible se resserrent. Les auteures notent que « parmi ceux qui ambitionnent un métier qui 
sache mettre en œuvre les potentialités et valoriser les compétences possédées, émergent surtout les mécontents: italiens poussés à tenter « l'aventure britannique », du fait de la difficulté expérimentée par les opportunités réduites d'insertion dans le monde du travail italien » (Bartolini \& Volpi 2005 : 103)[2].

Maria Immacolata Macioti, quant à elle, du fait d'une composition plus jeune et instruite de la population émigrée italienne en France, en appelle à oublier le concept d'assimilation pour parler d'intégration, qui implique selon elle « un respect des autres cultures sur un plan paritaire »[3] (Macioti 2005 : 301). Comment après cette remarque est-il possible d'occulter la question sociale ? Aux catégories sociales privilégiées le choix de leurs références, aux catégories sociales plus modestes leur inculcation ? Comme de nombreux chercheurs, elle décrit les italiens de la nouvelle émigration (en opposition avec ceux des vagues précédentes mus par la nécessité) comme des protagonistes, avec des niveaux moyens-hauts de préparation scolaire. Pourtant, l'image d'un voyageur international, indifférent aux lieux de travail et de vie, est, à notre sens, faussée par une idéologie qui voit l'individu comme maître absolu de son destin. Maria Immacolata Macioti nous rappelle d'ailleurs que tous les jeunes italiens en France aujourd'hui ne sont pas des managers ou entrepreneurs de renom, ni même des intellectuels de réputation internationale. La composition sociale de cette émigration a certes changé. Mais faut-il pour autant conclure à une disparition de la contrainte ? Le poids des structures sociales n'est pas amoindri, ce sont les formes des pressions, des injonctions qui se sont transformées. Pour simplifier, nous pourrions dire que l'aspiration au cosmopolitisme, à l'international, correspond à une volonté d'ascension sociale (l'élite lui étant souvent associée). Les classes moyennes et même certaines catégories populaires dont on méprise le «localisme », se trouvent aujourd'hui légitimement attirées par « l’international » qui procure une certaine reconnaissance sociale (Wagner 1998). Mais elles se trouvent aussi confrontées à la recomposition sociale des migrations et des rapports de force. Comme dans l'enseignement supérieur la croissance quantitative, l'augmentation du nombre d'étudiants mobiles ne garantit pas, en soi, la démocratisation de l'accès et de la réussite pour tous dans le monde professionnel.

Cet exemple montre que, selon la valorisation faite de l'objet ou du sujet, les chercheurs pencheront davantage vers la détermination ou l'indétermination. Si l'objet est valorisé, c'est la détermination qui primera et lorsque l'on mettra en avant le sujet, c'est la richesse, la liberté qui ressortira des analyses. Pourtant sujet et objet sont constitutifs l'un de l'autre. Ce qui importe donc c'est d'introduire des interstices dans la dualité, de s'interroger sur ce qui fait que l'on va déplacer la focale d'un coté ou de l'autre du continuum entre autonomie et contrainte pour permettent le dépassement des antagonismes. L'analyse mixte, comme la synthèse est donc intéressante, elle ne nie l'existence d’aucun aspect de la réalité.

\subsection{Sociologie pragmatique/sociologie militante}

Le dépassement de l'opposition entre acteur et système, entre autonomie et contrainte, passe aussi par le dépassement de l'opposition entre sociologie pragmatique et sociologie militante. Il n'y a jamais eu de passage d'une sociologie unifiée à une sociologie éclatée en paradigmes concurrents (Dubar 2006). Lorsque l'on observe la production sociologique à travers le temps et l'espace, on constate simplement des changements dans la structuration de la discipline et des modifications du poids des paradigmes différents, qui, de tout temps, ont structuré le champ de la sociologie. Depuis les pères fondateurs, les sociologues se sont toujours mutuellement reprochés d'être soit de brillants essayistes, trop militants, mais dont la pensée manque de rigueur systématique et d'unité soit d'être technicistes, rigoureux, mais sans visée analytique et interprétative (so, what ?). Le problème ici encore soulevé est selon les termes de Jean Michel Berthelot la capacité de la discipline à produire «l'intelligence de son objet » et celleci ne peut ni résider dans une vaste construction idéologique surplombante, ni dans l'accumulation technicienne de données (Berthelot 1990).

Les critiques adressées à une sociologie militante peuvent se résumer ainsi : certains chercheurs renfermeraient la théorie et les recherches sur elles-mêmes dans un doctrinisme ou un dogmatisme qui les pétrifient. Le militantisme ne doit pas être confondu avec l'idéologie, qui contrairement à une idée rependue de nos jours, à un sens tout à fait neutre. Une idéologie est un système d'idées. Le militantisme désigne l'ardeur dont font preuve certains acteurs du monde social en vue de rallier des personnes à une cause. Reste à définir la cause. La cause pouvant être la diffusion d'un savoir, le dévoilement d'un fonctionnement social ignoré, etc. Si l'on prend cette définition, alors tout chercheur qui produit et encadre des jeunes chercheurs semble être un militant, puisqu'il tente de les attirer dans sa sphère de réflexion et d'action. La sociologie, comme discipline qui tente d'échapper au sens commun, qui promeut la rigueur, la déconstruction, la transparence des procédures d'enquête, porte en elle-même un message, si ce n'est militant, au 
moins partisan. Le choix d'un sujet d'étude peut porter en lui la marque d'une militance. Choisir par exemple des sujets qui semblent ne porter la marque d'aucun doctrinisme et sans résonnance avec des politiques publiques est en soi un choix militant, qui porte la sociologie vers une marginalisation relative (Dubar 2006). Sans parler du choix des mots. Jean Claude Passeron (Passeron 1990), dans le raisonnement sociologique nous rappelle que les mots en sociologie sont utilisés comme des concepts polymorphes qui dérogent à l'univocité des sciences nomologique. Et l'indexation des mots au contexte historique, rend leur utilisation délicate d'un point de vue idéologique. L'usage d'un mot, d'une référence, d'un concept, d'un paradigme est un choix militant entre plusieurs conceptions du monde social. Les sociologues qui reprochent à leurs confrères leur militance, ne seraient-ils pas les premiers des militants ?

La critique du militantisme de certains sociologues se couple souvent d'un éloge des techniques de plus en plus sophistiquées, qui rendraient les analyses mécaniquement objectives, du fait de l'effacement du sujet qui analyse. On peut émettre des réserves sur cette sociologie techniciste, qui a certes l'avantage de faire des économies de temps, réduisant la durée des enquêtes de terrain in situ, mais a le désavantage de pousser à un tel réductionnisme, qu’il devient difficile pour l'analyste de passer de la description fine d'un phénomène, de constats statistiques, à l'interprétation. A moins de considérer évidemment que la sociologie est une science de la description, on ne peut se contenter de la sécurité de l'axiomatisation et de la puissance de l'outil mathématique, sans perdre de vue l'ouverture de «l'intelligence de l'objet» (Berthelot 1996 : 162). Les outils quantitatifs de description du social, la modélisation sont certes des instruments utilisables et à utiliser lorsqu'ils sont accompagnés d'une réflexion sur les modes de construction des catégories et des indicateurs statistiques, comme appui aux analyses sociologiques, mais toujours en arrière plan, afin de donner de l'espace et de l'ouverture argumentative nécessaire aux interprétations. « De plus en plus la mathématisation et la formulation ont désintégré les êtres et les expériences pour ne considérer comme seules réalités que les formules et équations gouvernant les entités quantifiées » écrit Edgar Morin (Morin 1990 : 19). Sous prétexte de mettre de l'ordre dans les phénomènes, de clarifier, de distinguer, en refoulant le désordre, l'incertain, ont normalise et on s'éloigne du vécu des individus. Ce sont certes des opérations nécessaires à l'intelligibilité, mais cela rend les interprétations difficiles. Le fait statistique ignore le sens. Il ne saisit que le caractère probabilitaireimprobabilitaire, non pas la structure des comportements et l'aspect organisationnel. Une simple analyse statistique n'est capable de comprendre ni la naissance, ni la croissance et l'orientation d'un phénomène. Ce qui ne signifie pas qu'il faille rejeter ces techniques, mais elles ne peuvent constituer en elle-même la preuve d'une scientificité et le cœur même de la discipline sociologique. Car une simple analyse statistique fait des phénomènes sociaux des entités de même nature que la matière, elle substantialise.

\subsection{Approche quantitative/approche qualitative}

Cette rivalité entre les tenants d'une sociologie pragmatique et les tenants d'une sociologie militante, s'accompagne souvent de controverses sur la fiabilité des méthodes de l'enquête sociologique. En résulte une opposition entre approche quantitative et approche qualitative, qui est aussi formelle que la distinction entre théories et méthodologies. Préalablement à tout travail de terrain, le sociologue élabore un cadre conceptuel, qui a comme fonction la centration, la délimitation du recueil de données. Il n'en est pas moins souple et ouvert. L'adoption d'une palette variée de méthodes d'investigation et d'un mode d'approche transversal permet de regarder un problème entier dans sa complexité comme une configuration ("look at the whole problem as a configuration ») en s'intéressant aux processus, aux dynamiques et développements plutôt qu'au statu quo, comme le souligne Piet J.M. Verschuren (Verschuren 2003). L'étude de cas, prise dans une perspective holistique, est en fait un choix d'organisation des données dans le but de préserver le caractère unitaire de l'objet social étudié. Piet J.M. Verschuren montre ainsi que « l'observation holistique », comme il la nomme, est basée sur deux piliers étroitement reliés entre eux :

- (a) le regard sur l'objet comme un tout

- (b) l'attitude «open-ended»

En sociologie, entre une attitude totalement ouverte en finalité et un programme de recherche hautement préstructuré, existe l'analyse mixte, la recherche itérative-parallèle, c'est-à-dire un mouvement continu d'aller-retour entre les diverses étapes de la recherche. Opposer les méthodes est donc vain, il s'agit en fait d'assurer la pertinence des méthodes, c'est-à dire de les ajuster aux questions posées et aux informations accessibles (Combessie 1999). Une compréhension approfondie des langues (qui comprend le langage de programmation des logiciel de traitement de données) et est aussi de rigueur dans toute recherche comparative, surtout lorsqu'elle est internationale. Comme nous le rappelle justement Pierre-Emmanuel Dauzat, lorsqu'on étudie une langue, on doit faire face «à la fois à un 
instrument, à une partition et à la musique [...] Pour ne pas commettre de fautes, il faut se barder de savoirs»[4]. Pour éviter les travers d'un comparatisme naif, il est important d'observer avec attention les modes de construction des catégories statistiques et notionnelles, veiller aux pièges de la traduction linguistique et être attentif aux principales dispositions juridiques qui encadrent l'objet étudié. Ce qui encourage à une immersion prolongée et répétée dans les lieux étudiés, sur le terrain.

L'enchaînement que Jean-Claude Combessie qualifie de «plus habituel » dans des « méthodes à vocation exploratoire », du type observation $\rightarrow$ entretien $\rightarrow$ questionnaire, ne semble pas toujours fécond. Il est beaucoup plus intéressant d'utiliser ces techniques simultanément, de manière à ce qu'elles s'enrichissent mutuellement, l'une appelant la modification de l'autre. Le regard et l'analyse s'en trouvent ainsi progressivement affinés. Il importe également de souligner que quelle que soit la méthode utilisée en sciences sociales, les données ne sont jamais recueillies, mais toujours construites (Eyraud 2008). Finalement c'est l'écriture qui permet de rendre toute démarche intelligible et de rétablir quelque peu l'unité à une réalité, que l'arsenal méthodologique a toujours tendance à morceler. Evoquer les «bricolages » et des doutes méthodologiques et théoriques rend la généralisation des résultats envisageable analytiquement. C'est-à-dire qu'il n'est en effet jamais possible de généraliser à l'ensemble de la population mère lorsqu'il s'agit d'une étude de cas, mais que par contre, il est possible et souhaitable d'avancer des propositions théoriques plus générales à partir de l'induction analytique. Comme nous le montre Daniel Bizeul, la communication des dispositifs et des opérations de l'enquête aide à faire la part des aspects circonstanciels, ainsi que des aspects stables et généraux dans les résultats d'analyse (Bizeul, 1998).

La fiabilité des données est donc un faux problème. Lorsque l'on travaille sur un terrain, on en a toujours une connaissance préalable, située socialement. Il semble donc peu pertinent de réduire les déterminations qualitatives à des déterminations quantitatives, comme il semble peu sérieux d'écarter les statistiques comme outils de compréhension du monde social. La sociologie ne peut se réduire à une géométrie du monde social[5]. Pourtant aujourd'hui du fait d'un usage croissant de techniques économétriques, se développent une sociologie que j'appellerai géométrique-illustrative, dont les techniques impressionnent et qui permettent des descriptions très intéressantes de la réalité sociale, mais qui pose des problèmes d'interprétation.

\section{Les conditions matérielles de la production sociologique}

$\mathrm{Au}$ cours de mes quasi-dix années d'expérience de recherche dans différentes universités, à observer l'enseignement de la sociologie comparativement dans plusieurs institutions de plusieurs pays, j’ai relevé, à l'instar d'autres chercheurs, les divergences existantes dans la transmission et l'évaluation des savoirs (Vitale, 2005). La diversité est intra-nationale, intra-européenne et intercontinentale. Comparer ces enseignements permet d'observer les divers courants et angles d'approche qui traversent la sociologie. Certains par des effets de mode ou d'opportunisme, (largement liés au contexte social), se réactivent et d'autres sont délaissés. Comme dans la nature, en sociologie, rien ne se crée, rien ne se perd, tout se transforme. «L'histoire de la discipline montre qu'en un siècle la construction de son savoir s'est effectuée sur le mode de la spécialisation et de l'hybridation des théories, des modes d'approche et des objets. Le vieux rêve nomologique de la discipline n'a cessé de se heurter au relativisme scientifique qui séduit aujourd'hui tant les sociologues. Il n'existerait ainsi pas une mais des sociologies, qui ne peuvent s'inscrire dans un même paradigme tant leurs langages sont incommensurables.» (Vitale, 2005, p.11) Pourtant derrière «l'anomie de surface», il y a bien un tronc commun, un objet, un horizon épistémique/épistémologique et de la rigueur scientifique (Vitale, 2005, p.13, Dubar, 2006).

\subsection{Droit à l'existence et « prix du temps »}

«La connaissance du social a mauvaise presse » (Berthelot 1990 : 13) ? Est-ce toujours d'actualité, en particulier depuis l'institutionnalisation de la discipline et la multiplication des sollicitations de plus en plus pressante du monde politique et social ? Dans le développement de la sociologie on prend rarement en considération les innombrables influences de l'environnement matériel, politique et social. Pourtant, bien plus que la plupart des sciences aux fondements bien établis, les sciences de la société se trouvent dans la situation défavorable d'avoir à prouver leurs droits à l'existence (Legrand, Guillaume, Vranken, 1995). Faut-il prendre au sérieux les critiques adressées à la sociologie et ce contexte de suspicion ? De quoi se nourrit-il ? Est-il fondé ? Il s'alimente, entre autres, d'études sociologiques. Parce qu'ils évoquent le relativisme, la difficile objectivité, parce qu'ils proclament l'arbitraire et la 
pluralité des savoirs et de leur transmission, les sociologues doivent sans cesse justifier leur démarche, la rendre transparente.

Les problèmes liés au statut épistémologique et cognitif de la sociologie se doublent d'une légitimité académique et sociale inconfortable, qui l'oblige à réfléchir souvent à ses fondements et plus largement au métier de sociologue. Pourtant la sociologie attire de nombreux étudiants, mais au lieu de voir dans ce phénomène le sceau de la force, on y voit un signe de faiblesse. Je pourrais citer, comme Philippe Vitale au Claude Dubar, les «affaires » Sokal aux EtatsUnis et Teissier en France, qui ont entamé fortement la crédibilité scientifique de la discipline (Vitale 2005, Dubar 2006). Mais « l'affaire Teissier »[6] par exemple révèle en fait les problèmes récurrents d'encadrement et de suivi des recherches doctorales au sein des universités. Le nombre de doctorants (notamment non-financés) sous la direction d'un même professeur est un signe qui ne trompe pas. Dans l'enquête de Roman Vourc'h sur les doctorants français, leur profil et leurs conditions de formation, ceux dit «professionnels », qui bénéficient d'un financement et travaillent souvent dans leur université de rattachement sont pour trois quarts inscrits en sciences. Ils sont également plus jeunes que la moyenne et moins isolés que les doctorants en SHS, qui déclarent en majorité travailler seuls (Vourc'h, 2010). Les doctorants financés, qui affirment bénéficier, au moment de l'enquête, de ressources issues d'un travail « intégré » à leurs études et/ou d'une allocation d'études directement liée à la réalisation de la thèse représentent 56,4\% de l'ensemble. Les situations diffèrent significativement selon les disciplines puisque près de neuf doctorants sur dix sont financés en sciences, contre moins d'un sur trois en lettres-SHS. 31,3\% des doctorants sont salariés, c'est-à-dire qu'ils travaillent «à côté» de leurs études Il s'agit d'une situation très fréquente parmi les doctorants en lettres-SHS puisque $52 \%$ d'entre eux réalisent leur thèse en étant salariés (les deux tiers sont enseignants). Très peu représentés en sciences, les doctorants sans financements regroupent environ un doctorant sur cinq dans les autres champs disciplinaires (12,3\% de l'ensemble). Ne percevant pas de ressources issues d'une activité rémunérée (en lien ou non avec leur travail de recherche) ou d'une allocation d'études, ils bénéficient plus fréquemment d'une aide directe de leur famille (Vourc'h 2010). Les sciences de la société sont «sous-dotées » en matière d'allocations ministérielles de recherche et de financement public et privé. De même, les écarts d’âge au recrutement des maitres de conférences entre SHS (sciences humaines et sociales) et SSM (sciences et structure de la matière) sont en moyenne de 6 ans. En 2004, l'âge moyen des maîtres de conférences recrutés (articles 26-I-1, 26-I-2 et 26-I-3, hors opérations de mutation et détachement) était de 32 ans et 8 mois[7]. Cette moyenne cache néanmoins des écarts entre grandes disciplines : pour les «sciences» (groupes 5 à 11 des sections CNU) l'âge moyen était de 30 ans et 7 mois, pour le droit, économie, gestion (groupes 1 et 2), de 31 ans et 9 mois et pour les lettres, langues, sciences humaines et sociales (groupes 3, 4, 12) de 36 ans et 7 mois.

Comment comprendre ces différences entre les disciplines en matière de financement ? La suspicion d'inutilité ? La production sociologique ne serait pas immédiatement palpables. Je suggérais aussi l'indiscipline de la discipline, son aspect décapant, dérangeant. Les facteurs sont multiples. Toujours est-il que les chercheurs en sociologie des universités ne produisent pas dans les mêmes conditions que les chercheurs de beaucoup d'autres disciplines. Leurs parcours d'insertion professionnelle, très souvent chaotiques, ont un effet au moins indirect sur leur production scientifique. La variabilité de la production sociologique est aussi variabilité des lieux de la production. Pour comprendre cette dernière il serait donc opportun, à la fois d'avoir un découpage disciplinaire et géographique plus fin, mais aussi de rentrer dans les méandres des institutions productives. Le système dual français par exemple produit des «types» de sociologues différents. Non pas parce qu'il existerait des natures, des tempéraments naturellement variables chez les sociologues, mais parce qu'ils sont, en autres, les produits de leurs conditions de production. Dans ces dernières joue un élément important : le temps. Le temps que possède un chercheur pour faire de la recherche ne se mesure pas simplement à l'aide d'un ou deux indicateurs. Étudier le « prix » du temps demande une incursion dans divers domaines de la vie sociale, institutionnelle, professionnelle et familiale des chercheurs.

Comment comprendre en sociologie la multiplication de l'intérêt pour les " petits objets ", la décontextualisation des analyses, la mosaïque de travaux ? Le développement d'une sociologie géométrique-illustrative, dont je parlais précédemment, est allé de pair avec une réduction du temps disponible pour lire, s’imprégner, faire du terrain et avec la multiplication des contrats courts financés. Les jeunes sociologues par ce biais, développent de plus en plus une capacité à « s'accrocher » ici et là, au fil des contrats à durée déterminée qu’ils décrochent, à un jeu de références disciplinaires, à mobiliser ou démobiliser, à s'ouvrir ou à mettre de coté des « lignes ou des points de saillance significatifs » selon les termes de Jean Michel Berthelot, suivant la demande sociale. Chaque sociologue s'engage dans une aventure intellectuelle certes personnelle, mais contrainte par les cadres institutionnels d'exercice (Berthelot 1996). La sociologie se conjugue au pluriel, parce qu'il existe à la fois des intermittents, des précaires, des « statutaires

(C) 2010 Émulations - Revue des jeunes chercheurs en sciences sociales

www.revue-emulations.net 
» et des « émérites » de la sociologie. La première difficulté lorsqu'un sociologue s'attèle à l'étude de l’intelligence du social est le manque de temps dont il dispose pour le faire et surtout en saisir les évolutions. Paul Ricoeur dans De l'interprétation, nous rappelle, à propos de Freud, " qu'il n'avait pas atteint du premier coup une vue claire de l'enchevêtrement des points de vue dans la métapsychologie » (Ricoeur 1965 : 76). Le temps qu'un scientifique peut consacrer à sa recherche et à l'écriture, dépend non seulement de son institution d'appartenance, des activités d'enseignement et responsabilités collectives qu'il a par ailleurs, mais également de son environnement familial et matériel. Le choix de l'analyse comparative internationale par exemple est un engagement nécessitant un investissement très important en temps, en déplacement, il a des conséquences sur la vie hors travail, et cette dernière en retour a des conséquences sur la production scientifique (Ackers 2010).

\subsection{Les sociologues : une catégorie en mutation}

Selon les données du CEREQ reprises par Odile Piriou en moyenne 150 thèses par an sont produites en sociologie en France, or depuis 2003 le secteur de la sociologie " académique » offre en moyenne 42 postes de jeunes chercheurs par an. Deux tiers des docteurs en sociologie doivent chaque année prospecter un emploi en dehors du secteur académique. Et même si plus généralement, en 2004, 47\% des docteurs en Sciences Humaines et Sociales intègrent l'Université et la recherche, $27 \%$ d'entre eux sont sur des statuts précaires ou temporaires (contractuels, ATER, Vacataires, etc.). Aujourd'hui dans les laboratoires associés au CNRS, en Ile de France, la population de personnels permanents représente en moyenne 39\% du total des membres (Piriou 2008).

L'ensemble des études s'intéressant au recrutement et carrières post-thèse en SHS soulignent la diversité des parcours suivant les filières. Les thèses en SHS durent plus longtemps que celles en « sciences exactes » (Piriou 2008 , Giret 2005 , Beret, Giret \& Recotillet 2004). Matthieu Hély et Sophie Pochic avancent plusieurs facteurs explicatifs : le faible taux de financement, une forte pratique d'enseignement considérée comme expérience professionnelle et enfin une expérience plus solitaire et un faible encadrement collectif (Vourc'h 2010 , Hély \& Pochic 2008). On pourrait aussi ajouter une conception de la thèse, en France, héritée de l'époque des thèses d'état, qui concevait l'exercice comme l'œuvre d'une vie et non comme une première expérience professionnelle de recherche. Et cette conception, comme l'absence fréquente d'un collectif qui encadre, pèse directement sur l'image d'une discipline quelquefois justement suspectée de fabriquer des intellectuels au relationnel difficile et/ou à l'égocentrisme exacerbé. Les tentatives actuelles d'harmonisation autour d'un modèle européen de la thèse en trois ans, qui fait du doctorat une première expérience de recherche[8], se heurtent à des pratiques et conceptions anciennes dans les départements en SHS, même si la tendance appliquée de professionnalisation touche aussi le secteur académique de la sociologie, qui suit ce qu'Odile Piriou appelle le « tournant praticien », accéléré par les reformes actuelles de l'enseignement supérieur et de la recherche. Les jeunes chercheurs des universités françaises doivent ainsi répondre à une triple injonction contradictoire, celle de leurs collègues, qui leur demandent de s'investir dans les tâches d'enseignement et d'encadrement, celle de leur directeur, qui leur demandent de fournir un travail d'enquête probant et un travail réflexif qui requiert recul et temps, et celle provenant de l'administration, qui suit les recommandations de politiques nationales et supranationales, qui incitent à la rapidité. Tout ceci pèse sur les résultats de la production scientifique.

Comme tout acteur, le sociologue se meut dans un environnement économique et social qui le contraint. Dès la formation doctorale, les parcours des sociologues se différencient. Aujourd'hui, du fait de la massification des universités, tous les doctorants ne sont pas des " héritiers ", ils ne possèdent pas tous non plus un soutien familial sans condition. Et le doctorat n'est qu'une première étape dans une formation, voire une carrière d'enseignantchercheur. Le temps dont dispose un maitre de conférence pour la recherche est là aussi variable suivant son institution d'appartenance, son sexe, son âge. On est loin d'un groupe social homogène. Pas plus que les carrières internationales ne correspondent à des stratégies unilatérales d'ascension professionnelle, les sociologues ne forment un « international » homogène sur le plan de leurs intérêts comme de leurs pratiques (Wagner 1998). La production sociologique dépend aussi de la présence plus ou moins importante, au sein des universités et des laboratoires d'une communauté disciplinaire solide et solidaire. Elle dépend également des formes de travail collectif et d'un modèle, d'une structure institutionnelle, historiquement constitué. Comment, dans ces conditions et face aux évolutions précédemment évoquées, ne pas s’interroger sur les producteurs de la recherche sociologique ?

Réfléchir sur les problèmes de la relation du chercheur de terrain avec son milieu n'est pas nouveau. On assiste depuis plusieurs décennies en Europe à «l'inflation » des diplômes, à l'augmentation des effectifs en première cycle et au changement du métier d'enseignant-chercheur dont les tâches d'accompagnement pédagogique et 
administratives pèsent de plus en plus sur la recherche (Duru-Bellat 2006). Le développement de l'enseignement et de la recherche sociologique se réalise dans un contexte d'autonomisation des universités. La segmentation institutionnelle s'accélère. Les conditions d'enseignement et de recherche, la reconnaissance institutionnelle, les dotations financières, d'un département à l'autre, d'un laboratoire à l'autre varient énormément. Les docteurs se trouvent devant un marché du travail académique de plus en plus concurrentiel, mais ils ne possèdent pas tous les mêmes «atouts ", ils n'ont pas toujours les moyens de construire une pensée et des réseaux sociaux à la hauteur de leurs ambitions. Comme Matthieu Hély et Sophie Pochic le soulignent, après leur thèse certains docteurs se trouvent à préparer les concours de la fonction publique, dont ceux de l'enseignement avec le « sentiment amer d'avoir perdu leur temps » (Hély \& Pochic 2008). D’autres cumulent les post-doctorats. Ces derniers en effet se multiplient en raison de l'augmentation du nombre de docteurs, de la concurrence accrue intra et internationale entre jeunes chercheurs et de la stagnation, voir de la diminution du nombre de postes stables et permanents. L'institutionnalisation du postdoctorat tend à élever de plus en plus le niveau d'exigence requis, en termes de publications en particulier, pour obtenir un contrat stable. En SHS ces contrats courts, ces emplois d'attente, voir précaires sont proposés à des « jeunes » qui ont souvent largement dépassé la trentaine en France (et quelque fois la quarantaine en Italie !) Le passage du local au national, puis à l'international n'estompe pas la segmentation et la spécialisation des parcours des docteurs, bien au contraire, il les complique et ce faisant il fragmente encore davantage la discipline.

Les sociologues sont formés par des institutions historiquement construites, qui s’insèrent dans des tissus économiques et sociaux particuliers en Europe. Chaque chercheur fait partie d'un segment particulier et différencié de l'enseignement supérieur européen (directement en concurrence avec d'autres segments). D'où l'intérêt de s'interroger sur son statut et son rang. Et d'abandonner le point de vue d'une sorte de trône supérieur d'où contempler la société. Il n'y a d'objet que par rapport à un sujet (qui observe, isole, définit, pense). Une certaine « exhibition » de sa place par rapport à ce que l'on étudie, comporte de l'humilité (Morin 1990 : 11). L'honnêteté intellectuelle passe certes facilement pour de l'incompétence, mais c'est aussi une marque de modestie, qualité qui me semble indispensable pour faire de la recherche. Les versions du monde sont autant de schèmes de lecture et d'interprétation qui reposent, notamment, sur un filtre culturel et anthropologique. Lorsque transparaît le subjectif, cela donne au lecteur la possibilité de le détecter. Il est donc plus honnête intellectuellement de prôner le retour de l'observateur dans les sciences humaines et sociales plutôt que de se cacher derrière un « nous » ou un « on », qui n'épargne en rien les prises de position idéologiques. L'observateur n'étant pas exactement le sujet, il est un sujet collectif : Qui fait la recherche? Qui a formé ce chercheur? Qui est le commanditaire de l'étude ? etc.

Dans les travaux de Simona Bartolini et Frederica Volpi déjà cités, les propos des interviewés sont rapportés en ces termes : "Selon ces jeunes, il manque dans la culture italienne une approche du travail qui se fonde sur une distribution juste des mérites et de la responsabilité [...] À leurs yeux, en fait, le Royaume-Uni se présente comme un Eldorado voisin, dans lequel chacun a la possibilité d'exploiter des opportunités professionnelles sérieuses et des revenus faciles, de capitaliser les expériences et de se faire apprécier pour leur œuvre [9]» (Bartolini Volpi : 2005). Ces opinions, selon ces auteures ne s'appuient pas sur des « expériences concrètes ", mais sur une présomption d'une « incapacité générale » du marché du travail italien à créer des conditions idéales pour l'exercice de leurs professions. Elles parlent également d'abandon de la mère patrie « abbandonare la madrepatria ». Ce qui est mouvant, nous disentelles, est par définition fuyant[10], l’identité des jeunes italiens en Angleterre réside dans le transitoire «transitorietà ». L'italianité « l'italianità », devient une question personnelle. Elles voient alors des individus qui habitent un village global «villaggio globale » en construction et les présentent comme mus par un individualisme effréné, en ne prenant jamais au sérieux les critiques qu'ils adressent à leur pays natal. Le fondement de ces critiques serait pourtant à étudier. Ces interprétations peuvent être néanmoins comprises, au regard de la politique actuelle et du commanditaire de cette recherche. Cet article est, en effet, une contribution à un ouvrage collectif qui a été réalisé avec le concours du Ministère des affaires étrangères italiennes et du Conseil général des italiens à l'étranger. Il serait particulièrement intéressant de mettre en parallèle des informations sur la formation du sociologue qui écrit, sur le commanditaire et le sujet qu'il aborde. Cette réintégration de l'observateur dans son observation permettrait de suivre les évolutions d'une catégorie socioprofessionnelle fragmentée. 


\section{Conclusion}

Si le thème de cet article est ambitieux et risqué, mon propos aspire à la modestie. Je souhaitais simplement ici reformuler et apporter une pierre à d'édifice déjà solide de l'ensemble des réflexions existantes sur le sujet. Cet article est une incitation à s'intéresser collectivement à nos propres parcours et pratiques professionnelles, aux cadres paradigmatiques, qui définissent l'activité de connaissance et les procédures concrètes qui la réalisent. J'espère ainsi nourrir le débat sur des questions anciennes en sociologie et ce faisant rappeler, comme bien d'autres l'on fait avant moi, que par delà ses clivages de surface, les conflits et les discontinuités dans les carrières des enseignantschercheurs, la sociologie s'organise bien selon « un maillage cognitif », une toile, " des lignes de force » (Berthelot 1990). Elle connait certes une division interne, qui relève à la fois de la segmentation en champs, mais aussi de la mise en œuvre de « points de vue», d'approches différentes. Mais on pourrait se demander comme Philippe Vitale s’il n'existerait pas un modèle fédéraliste au cœur même de la discipline sociologique, de son épistémologie, de ses langages, de ses théories et méthodes (Vitale 2005 : 197). Ce qui ne signifierait pas que les travaux sociologiques soient des fourre-tout, des empilements, car mis bout à bout, on lit aisément une logique épistémologique, une articulation, un tronc commun. Dans la pluralité des dimensions étudiées lors d'une enquête sociologique, il ne faut pas voir la marque de l'éclectisme, mais l'intelligence de l'objet qui se manifeste par un maquis construit par différents courants et terminologies (Berthelot 1990).

Ce maquis est aussi un construit naissant des conditions diversifiées de la production sociologique et la multiplication du nombre d'inscrits et de diplômés de la discipline, qui n'ont cessé d'alimenter la segmentation de cette dernière. Les espaces-temps institutionnels traversés par les chercheurs, les financements obtenus et les sujets d'actualité traités, sont également sans cesse renouvelés par la demande sociale. Cependant, la plupart des sociologues parviennent à concilier le vecteur de l'activité scientifique (construire une explication rationnelle et empiriquement fondée du monde), les aléas empiriques de cette construction et le contexte historique et social de l'activité. Il est donc nécessaire de rejeter l'idée d’une culture (romanesque) sociologique sacralisée, anhistorique et désintéressée, comme de rappeler la nécessaire autonomie des sociologues face à l'instrumentalisme économique et politique et réorienter le débat théorique vers une analyse rhizomique pour reprendre les termes de Berthelot (i.e : étudier le jeu de la justification ou de son absence) des producteurs et des relais du savoir sociologique. Il me semble en effet important que la sociologie reste « une sorte de réservoir un peu brouillon et anarchique, mais particulièrement fécond, d'outils analytiques et cognitifs où chacun peut puiser à son gré (...) seule discipline à simultanément couvrir la totalité du monde vécu et user de la totalité des grands outils cognitifs (...) elle est une sorte de laboratoire permanent de l'intelligence de l'objet en sciences sociales » (Berthelot 1996 : 164).

Dépasser la sociologie techniciste, comme dépasser la dérive des évaluations strictement quantitatives des laboratoires, c'est aussi mieux comprendre la richesse d'une discipline, qui s'attache aux régularités comme aux incertitudes et aux contingences (Bertaux 2008). La sociologie est faite de recherches nécessairement fragmentaires, mais ne doit pas se contenter de simples juxtapositions. Penser ensemble les individus, les interactions, les logiques sociales et culturelles, les contraintes et les marges de manœuvre possibles, est un exercice certes difficile, conflictuel, mais indispensable à la connaissance.

\section{Bibliographie}

ACKERS, Louise (2010), "Internationalisation and Equality. The Contribution of Short Stay Mobility to Progression in Science Careers" Recherches sociologiques et anthropologiques, $\mathrm{n}^{\circ} 1$.

BARTOLINI Simona, VOLPI Federica (2005), "Paradossi d'oltremanica. I giovani italiani in Inghilterra" , p91-116 In Caltabiano C., Gianturco G., Giovani oltre confine. I discendenti e gli epigoni dell'emigrazione italiana nel mondo, Roma, Carocci editore.

BERET Pierre, GIRET Jean François, RECOTILLET Isabelle (2004), «L'évolution des débouchés professionnels des docteurs : les enseignements de trois enquêtes du CEREQ », Education et formation, mars n67 p109-116

BERTAUX Daniel (2010), "Peut-on construire des indicateurs de notoriété des centres de sociologie ? », Socio-logos. Revue de l'association française de sociologie [En ligne], 3 | 2008, mis en ligne le 04 avril 2008, Consulté le 05 juillet 2010. URL : http:// socio-logos.revues.org/1703 
BERTHELOT Jean Michel (1996), Les vertus de l'incertitude, le travail de l'analyse dans les sciences sociale, PUF, Paris.

BERTHELOT Jean Michel (1990), L'intelligence du sociale, PUF, Paris.

BISSERET Noel (1974), Les inégaux on la sélection universitaire, PUF, 1974

BIZEUL Daniel (1998), "Le récit des conditions d'enquête : Exploiter l'information en connaissance de cause », RFS. vol. 39, no4, pp. 751-787

BIZEUL Daniel (2008), «Les sociologues ont-ils des comptes à rendre ? », Sociétés contemporaines, nº70, p.95-114

BLANCHET Alain (1987), Les techniques d'enquête en sciences sociales. Observer, interviewer, questionner, Dunod.

CALTABIANO Cristiano, GIANTURCO Giovanna (2005), Giovani oltre confine, I discendenti e gli epigoni dell'emigrażione italiana nel mondo, Roma, Carocci.

COMBESSIE, Jean Claude (1996), La méthode en sociologie, Paris, La découverte.

DUBET François (1994), Sociologie de l'expérience, Paris : Le seuil

DURU-BELLAT Marie (2002), L’inflation scolaire : les désillusions de la méritocratie, Paris, Seuil, 2006.

DUBAR Claude (2006), «Le pluralisme en sociologie : fondements, limites, enjeux », Socio-logos, Revue de l'association française de sociologie, Numéro 1, Varia.

EYRAUD Corine (2008), Les données en sciences sociales. Du matériau brut à la connaissance des phénomènes sociaux, Paris Armand Colin, Cursus Sociologie.

FRIEDBERG Erhard, VIDAL Daniel, DUBET François (1996), «Symposium sur sociologie de l'expérience ». Sociologie du travail, $\mathrm{n}^{\circ} 1 / 96, \mathrm{p} .81-100$

GIRET Jean-François (2005), De la thèse à l'emploi : les débuts professionnels des jeunes titulaires d'un doctorat, Marseille : CEREQ. Bref n²20, ISSN :0758-1858.

GRAWITZ Madeleine (1993), Méthodes des sciences sociales, éd. Dalloz.

HELY Matthieu, POCHIC Sophie (2008), «Recrutement et carrière post-thèse en SHS : Des parcours diversifiés », $V$ ie de la recherche scientifique, $\mathrm{n}^{\circ} 372, \mathrm{p} .24-26$

LEGRAND M., GUILLAUME J.F., VRANCKEN D (1995), La sociologie et ses métiers, l'Harmattan, Collection "Logique Sociales".

MACIOTI Maria Immacolata (2005), «La collettività italiana in Francia: un'emigrazione d”'élite” », in Caltabiano C., Gianturco G., Giovani oltre confine. I discendenti e gli epigoni dell'emigrazione italiana nel mondo, Carocci Roma.

PASCAL Blaise (1670), Pensées sur la religion, Paris.

PIRIOU O (2008), « Le nouveau tournant de la sociologie en France dans les années $2000 »$,Sociologies pratiques, $\mathrm{n}^{\circ}$ 16, mars, pp. 123-130.

PIRIOU O (2008), "Que deviennent les diplômés de sociologie ? Un état », Socio-logos, $\mathrm{N}^{\circ}$ 3, [En ligne] 4/2008.http://socio-logos.revues.org/1622

VALLEE Robert (1995), Cognition et système, essai d'épistémo-praxéologie, Lyon-Limonest, L'Interdisciplinaire.

PASSERON Jean-Claude (1990), Le raisonnement sociologique : l'espace non poppérien du raisonnement naturel, Paris : Nathan, Essais et Recherches.

RICOEUR Paul (1965), De l'interprétation, essai sur Freud, Paris, Seuil.

VERSCHUREN Piet J.M (2003), “ Case study as a research strategy: some ambiguities and opportunities”. International Journal of Social Research Methodology, Vol. 6 n² April-June.

VITALE Philippe (2005), La sociologie et son enseignement. Curricula, théories et recherches, Paris, L'Harmattan.

(C) 2010 Émulations - Revue des jeunes chercheurs en sciences sociales

www.revue-emulations.net 
VOURC’H Roman (2010), «Les doctorants. Profils et conditions d'études », OVE Infos n²4, Paris, Juin 2010

WAGNER Anne-Catherine (1998), Les nouvelles élites de la mondialisation. Une immigration dorée en France, Paris, PUF.

\section{Notes}

[1] p93. «I secondi sono invece espressione della nuova emigrazione e, quindi, artefici della scelta più autonoma (rispetto al passato) di vivere all'estero.»

[2] p103 «tra coloro che ambiscono a un'occupazione che sappua mettere a frutto le potenzialità e valorizzare le competenze possedute emergono sopratutto gli scontenti : italiani spinti a tenatre "l'avventura britannica", a motivo del disagio sperimentato per le ridotte opportunità d'inserimento nel mondo del lavoro italiano".

[3] Mocioti p301 «Per gli italiani presenti oggi in Francia si dovrebbe parlare di integrazione : concetto diverso da quello di assimilazione, che dovrebbe implicare il rispetto delle culture altre, accettate su piano paritario. »

[4] Les Mots de l'esprit, In Télérama, n²846, 28 juillet 2004

[5] Géométrie est ici entendue dans le sens de présentation du contenu de phénomènes sociaux par des formes, des points, des lignes, etc.

[6] Mme Teissier, astrologue-voyante, a soutenu une thèse de sociologie à la Sorbonne sous la direction de Monsieur Maffesoli. Un comité de relecture de cette thèse conclut au caractère non sociologique d'un manuscrit de 900 pages qui avait pourtant reçu une mention « très honorable ».

[7] Le bilan de la campagne 2004 de recrutement et d'affectation est disponible sur la Toile : http://www.education.gouv.fr/personnel/enseignant_superieur/enseignant_chercheur/statistiques.htm [8] Cf. European Charter for Researchers and a Code of Conduct for the Recruitment of Researchers http://ec.europa.eu/eracareers/pdf/am509774CEE EN E4.pdf

[9] «Secondo questi giovani, manca nella cultura italiana un approccio al lavoro che si fondi su una giusta distribuzione dei meriti e delle responsabilità[...] A loro occhi, infatti, il Regno Unito si presenta come un vicino Eldorado, in cui ciascuno ha modo di sfruttare opportunità professionali serie e facili guadagni, di capitalizzare esperienze e di farsi apprezzare per il proprio operato. »

[10] p104. «Cio’ che si nuove è sfuggente per definizione” 